\title{
Long period variables and mass loss in the globular clusters NGC 362 and NGC 2808
}

\author{
T. Lebzelter ${ }^{1}$ and P. R. Wood ${ }^{2}$ \\ ${ }^{1}$ Department of Astronomy, University of Vienna, Tuerkenschanzstrasse 17, 1180 Vienna, Austria \\ e-mail: thomas.lebzelter@univie.ac.at \\ 2 Research School of Astronomy and Astrophysics, The Australian National University, Canberra, Australia
}

Received 14 December 2010 / Accepted 21 March 2011

\begin{abstract}
Context. The pulsation periods of long period variables (LPVs) depend on their mass and helium abundance as well as on their luminosity and metal abundance. Comparison of the observed periods of LPVs in globular clusters with models is capable of revealing the amount of mass lost on the giant branch and the helium abundance.

Aims. We aim to determine the amount of mass loss that has occurred on the giant branches of the low metallicity globular clusters NGC 362 and NGC 2808. We also aim to see if the LPVs in NGC 2808 can tell us about helium abundance variations in this cluster. Methods. We have used optical monitoring of NGC 362 and NGC 2808 to determine periods for the LPVs in these clusters. We have made linear pulsation models for the pulsating stars in these clusters taking into account variations in mass and helium abundance. Results. Reliable periods have been determined for 11 LPVs in NGC 362 and 15 LPVs in NGC 2808. Comparison of the observed variables with models in the $\log P-K$ diagram shows that mass loss of $\sim 0.15-0.2 M_{\odot}$ is required on the first giant branch in these clusters, in agreement with estimates from other methods. In NGC 2808, there is evidence that a high helium abundance of $Y \sim 0.4$ is required to explain the periods of several of the LPVs.

Conclusions. It would be interesting to determine periods for LPVs in other Galactic globular clusters where a helium abundance variation is suspected to see if the completely independent test for a high helium abundance provided by the LPVs can confirm the high helium abundance estimates.
\end{abstract}

Key words. stars: AGB and post-AGB - globular clusters: individual: NGC 362 - globular clusters: individual: NGC 2808 stars: mass-loss - stars: variables: general

\section{Introduction}

Long period variability is a typical characteristic of low and intermediate mass stars during their evolution on the asymptotic giant branch (AGB). This evolutionary phase is of high importance as it is accompanied by high mass loss rates effectively removing the outer layers of a star and returning its material to the interstellar medium. Knowledge of this mass loss process has a strong impact on our understanding of stellar and galactochemical evolution.

Mass loss and variability seem to be linked on the AGB, with stars having high mass loss rates also showing large-amplitude pulsation. The radial pulsation lifts substantial amounts of material to the grain formation radius after which the radiation pressure force acting on the grains can drive a stellar wind (e.g. Wood 1979; Höfner 2008). While this scenario is widely accepted nowadays, the details of the mass loss process are not totally understood yet. This is partly due to the fact that data on stars with a well determined mass and metallicity are rare. Consequently, the dependency of mass loss on these two parameters could not be studied sufficiently in the past.

While it is very difficult to derive these basic parameters for field stars, the rather homogeneous data sets provided by globular clusters offer the possibility to work on this problem. We can safely assume that all stars we see at a given moment on the AGB of a cluster do not differ significantly in their mass and age or in their metallicity (although recent studies indicate that more than one episode of star formation may have occured in some globular clusters of the Milky Way, e.g. D'Antona \& Ventura 2008). Therefore the upper giant branches of globular clusters are an excellent testbed to study the properties of evolved stars.

Wood et al. (1999) have shown that long period variables (LPVs) form a number of parallel sequences in the periodluminosity diagram and that some of these sequences can be explained by radial pulsation in the fundamental mode and in low order overtone modes (see Soszyński et al. 2009; and Wood $\&$ Arnett 2010, for recent observational and theoretical versions of the period luminosity sequences for LPVs). Once a star starts to lose mass at a high rate, its total mass will start to decrease significantly, which will affect the observed period-luminosity sequences. Lebzelter \& Wood (2005) measured the $\log P-K$ sequences for the LPVs in the globular cluster 47 Tuc and they found that the shape of these sequences can only be explained if a steady decrease of mass on the RGB and the AGB is assumed. Their paper illustrated the possibility to investigate the mass loss rate of low mass stars by the study of an ensemble of AGB variables in a single stellar population.

The aim of the present paper is to extend this study to two further clusters with a metallicity different from that of 47 Tuc. The two clusters are NGC 362 and NGC 2808. In the following we will give a brief review of the literature on these two targets. Then we describe the observations obtained for this study in order to make a detailed search for LPVs. The number of previously known LPVs is small and not sufficient to define the path of $\log P-K$ sequences. Finally, we present the $\log P-K$ diagram 
for the two clusters and discuss it in the light of linear pulsation models and a Reimers-like mass loss rate.

\subsection{NGC 362}

With the publication of the first colour-magnitude diagram of the cluster by Menzies (1967), it became obvious that NGC 362 does not follow the standard picture of the cluster metallicity vs. colour-magnitude diagram (CMD) morphology. The horizontal branch, which is populated mainly on the red side of the instability strip, would suggest a comparatively high metallicity (according to Carretta \& Gratton 1997 similar to 47 Tuc, which would mean $[\mathrm{Fe} / \mathrm{H}]=-0.66$ ), while the slope of the giant branch, the integrated colours and the cluster's spectral type indicate a lower metallicity (e.g. Alcaino 1976; Harris 1982). The discrepancy appearing for this cluster provided a major input for the discussion of the 2 nd parameter problem in globular clusters (e.g. Alcaino 1976).

Several authors have published abundance studies for individual stars in NGC 362. Pilachowski (1981) derived a mean $[\mathrm{Fe} / \mathrm{H}]=-0.9$ from several cluster giants. Gratton (1987) measured a value of $[\mathrm{Fe} / \mathrm{H}]=-1.2$ from one star. Caldwell \& Dickens (1988) find $[\mathrm{Fe} / \mathrm{H}]=-0.9$ from 2 red giants. Shetrone $\&$ Keane $(2000)$ derive $[\mathrm{Fe} / \mathrm{H}]=-1.33 \pm 0.01$ from 12 giants in agreement with the work of Rutledge et al. (1997) giving -1.36. The compilation of Harris (1996) lists -1.16 based on earlier literature data. Photometric studies revealed a split of the cluster subgiants, RGB and AGB stars into a CN-rich and a CN-poor group, with the majority being CN-rich (Smith 1983, 1984). As a cause for this bimodality, Kayser et al. (2008) suggest an enrichment by intermediate mass AGB stars from a first generation of stars in the cluster (see also the summary on NGC 2808 given in the next section). The width of the main sequence, however, indicates that there is only a small spread in the overall metal abundance (Bolte 1987).

NGC 362 is thought to be slightly younger than other globular clusters, in particular it appears to be 1-3 Gyr younger than NGC 288, with which it is often compared due to the similar metallicity (e.g. Green \& Norris 1990; Catelan \& de Freitas Pacheco 1999). Gratton et al. (1997) give an age of 7.8 to $9.3 \mathrm{Gyr}$ for this cluster while Meissner \& Weiss (2006) find a an age of approximately $8.5 \pm 2$ Gyr.

\subsection{NGC 2808}

NGC 2808 is famous for various outstanding features in its CMD (Walker 1999; Bedin et al. 2000). Three main sequences have been identified (Piotto et al. 2007) which are attributed to three phases of star formation. The findings do not allow for an appreciable age difference between the three phases. In this context, the cluster's horizontal branch morphology is also quite puzzling, with a combination of a red clump-like structure and long tail blueward of the instability strip (Bedin et al. 2000; D'Antona \& Caloi 2008; Dalessandro et al. 2011). The number of RR Lyr stars is rather small (e.g. Clement \& Hazen 1989; Corwin et al. 2004).

In an attempt to explain these characteristics, it has been suggested that gas from which the second and third generation of stars formed has been contaminated by intermediate-mass AGB stars (D'Antona \& Caloi 2004, 2008). NGC 2808 is one of the clusters suspected to be massive enough to retain the AGB ejecta, thus He enriched material would be available for star formation. There is no spread in the iron abundance in the
Table 1. Adopted parameters for NGC 362 and NGC 2808.

\begin{tabular}{lccc}
\hline \hline & NGC 362 & NGC 2808 & NGC 104 \\
\hline$(m-M)_{V}$ & 14.80 & 15.56 & 13.50 \\
$E(B-V)$ & 0.05 & 0.23 & 0.02 \\
$M_{V, t}{ }^{a}$ & -8.43 & -9.39 & -9.42 \\
age $[\mathrm{yr}]$ & $10 \times 10^{9}$ & $10 \times 10^{9}$ & $11 \times 10^{9}$ \\
$Z$ & 0.001 & 0.001 & 0.004 \\
\hline
\end{tabular}

Notes. For comparison we also give the data for NGC 104 (see Lebzelter et al. 2005). ${ }^{(a)}$ Absolute visual magnitude from Harris (1996).

cluster, hence a He abundance spread from $Y \sim 0.25$ to 0.4 seems necessary to explain the triple main-sequence (MS) (Piotto et al. 2007). A further observation supporting this scenario comes from abundance measurements of red giants (Carretta et al. 2006) which show three groups of stars according to the Oabundance.

The alternative scenario trying to explain the HB morphology assumes an enhanced mass loss for a fraction of the red giants before reaching the tip of the red giant branch (RGB). Sandquist \& Martel (2007) found from the comparison of cumulative observed and synthetic luminosity functions that NGC 2808 shows a lack of stars immediately below the RGBtip, which they explain with this scenario.

Ferraro et al. (1990) give a summary on literature values for the metallicity and the reddening which range for $[\mathrm{Fe} / \mathrm{H}]$ from -0.96 to -1.48 , and for $E(B-V)$ from 0.21 to $0.34 \mathrm{mag}$, respectively. Rutledge et al. (1997) find $[\mathrm{Fe} / \mathrm{H}]=-1.34$ from spectroscopic observations of individual cluster members. Carretta et al. (2004), also from the analysis of individual stars, derive $[\mathrm{Fe} / \mathrm{H}]=-1.14 \pm 0.01$ on the Carretta \& Gratton scale corresponding to about -1.27 on the Zinn \& West scale. The compilation of Harris (1996) lists $[\mathrm{Fe} / \mathrm{H}]=-1.37$.

NGC 2808 is an old cluster with an age similar to 47 Tuc (Santos \& Piatti 2004). Quantitative age estimates scatter between 11 Gyr (D’Antona \& Caloi 2008) and 12.5 to $13 \mathrm{Gyr}$ (Piotto et al. 2007; D'Antona \& Caloi 2004). Somewhat younger is the determination by Meissner \& Weiss (2006) who give an age as low as 8 to 10 Gyr. In their detailed examination of globular cluster relative ages, Marin-Franch et al. (2009) found that NGC 2808 and NGC 362 had essentially indistinguishable ages which were about $15 \%$ smaller than the age of 47 Tuc. The parameters we have adopted for NGC 362 and NGC 2808 are given in Table 1.

\subsection{Previous searches for variable stars in our sample clusters}

As for most Milky Way globular clusters the searches for variable stars predominantly focused on variables on the horizontal branch or the main sequence and binaries. This has, in most cases, two consequences: first, many AGB stars are already saturated on the deep images required to reach the main sequence stars; and second, the monitoring typically focuses on time scales of days or weeks rather than the months or years which are needed to properly describe the variability of red giants. Therefore, as shown in Lebzelter \& Wood (2005) for the well studied cluster 47 Tuc, a lot of long period variables remained undiscovered.

However, in both NGC 362 and NGC 2808 a few long period variables have been discovered. For NGC 362 the Catalogue of Variable Stars in Globular Clusters (Clement et al. 2001) 
lists two candidates for LPVs, namely V2 (90 days period) and V16 (135 d). Concerning the period of V2 it has to be noted that an earlier time series by Sawyer (1931) led to a period of $105 \mathrm{~d}$, while the value of $90 \mathrm{~d}$ listed in Clement et al. (2001) stems from a short time series from Eggen (1972). Eggen noted this difference and attributed it to a possible period change that was also suspected by Sawyer-Hogg, as she could not fit a couple of early measurements of the star with the $105 \mathrm{~d}$ period. V2 is also remarkable due to its high $\mathrm{Li}$ abundance (Smith et al. 1999) indicating an ongoing extra-mixing process in this star. Data on the discovery and period of V16 have been summarized by Lloyd-Evans (1982). In the same paper it is shown that V16 has a high probability for cluster membership according to radial velocity data. The most recent detailed study has been published by Szekely et al. (2007). Beside V16 they detected four stars which they classified as long period variables, two of them are probably sources in the background. For one star, V17, they give a period of approximately $69 \mathrm{~d}$. The $K$-magnitude places the star on the RGB of NGC 362. As Szekely et al. note, their fourth LPV V36 is likely a cluster member according to the star's metallicity. $\mathrm{V} 2$ is not found in their list of variables.

After the discovery of the red variable V1 in NGC 2808 in the 1960s, Clement \& Hazen (1989) reported the finding of a second long period variable, V11, and presented first data on the light changes of these two stars. The variability of V1 has been confirmed by Corwin et al. (2004). For none of the LPVs could a period be derived.

\section{Observations and data analysis}

\subsection{Optical data}

The two clusters were monitored as part of a larger program searching for long period variables in Galactic globular clusters. Results for 47 Tuc (Lebzelter \& Wood 2005) and NGC 1846 (Lebzelter \& Wood 2007) have been published as the first papers from this monitoring program. The observations of NGC 2808 and NCG 362 were obtained and analysed the same way. Thus we will give here only a brief summary and refer to Lebzelter \& Wood (2005) for details.

The time series were obtained in two parts. The first part was observed at Mount Stromlo using the 50 inch telescope and the camera of the MACHO experiment (Alcock et al. 1992). The MACHO camera obtained two images in two broad band filters at the same time. These passbands did not correspond to standard filters but the blue one was similar to Johnson $V$. Observations started in May 2002, but came to an abrupt stop after a few months when Mount Stromlo observatory was destroyed by a bush fire.

We continued our monitoring program in late 2003 at CTIO's $1.3 \mathrm{~m}$ telescope operated by the SMARTS consortium. The instrument ANDICAM was used for two monitoring runs in service mode from October 2003 to January 2004 and from March 2005 to July 2005 (only NGC 2808), respectively. Monitoring was done in Johnson $V$ and $I_{\mathrm{C}}$.

The $V$ and the $I_{C}$ data sets were analyzed separately. To detect variable stars and to derive light curves we applied an image subtraction technique using the ISIS 2.1 code developed by Alard (2000). Stellar fluxes on the reference frames were measured using the PSF fitting software written by Ch. Alard for the DENIS project (see Schuller et al. 2003). A typical photometric accuracy derived from 2 images obtained in the same night is 0 . 02 in $V$.
For both clusters we observed two fields with ANDICAM, each having a field of view (FOV) of $6 \times 6$ arcmin. The central coordinates of the two fields had an offset of approximately 3 arcmin, i.e. the two fields were overlapping by almost $50 \%$. The field of the MACHO camera was considerably larger (even though we used only one segment out of 4), but no long period variables beyond the FOV covered by the ANDICAM images could be detected. For NGC 362, Rosenberg et al. (2000) give a core radius of 0.17 arcmin and a concentration parameter $\log \left(r_{\text {tidal }} / r_{\text {core }}\right)$ of 1.94 . For NGC 2808 corresponding values of 0.26 arcmin and 1.77 are listed. Accordingly, the tidal radius of both clusters is approximately 15 arcmin, and our observations, which have been centered at the cluster centre, extend to only 20 to $25 \%$ of the cluster's tidal radius. Since the stars of interest are relatively bright, they are individually uncrowded and we expect the giant branch sample of detected stars to be complete. In this region, the ratio of the cluster to the field star populations should be high.

To estimate the expected contamination by field stars in the direction of the two clusters we used the TRILEGAL model (Girardi et al. 2005) via its web interface ${ }^{1}$. Standard settings were applied including a halo contribution. Within a field of $0.02 \mathrm{deg}^{2}$ (the size of the two ANDICAM fields in each cluster) around the cluster centres the TRILEGAL output did not include any field stars that would by chance fall onto the upper giant branch in a $V$ vs. $V-I$ colour-magnitude diagram.

Among the variable stars detected, we selected the long period variables based on the brightness $(V<14.5$ for NGC 362, and $V<15$ for NGC 2808), timescale of the variation (exceeding 30 days) and a total light amplitude in $V$ of at least $0.1 \mathrm{mag}$. Period search was done using Period98 (Sperl 1998), a code which can compute a discrete Fourier transformation in combination with a least-squares fitting of multiple frequencies on the data. A maximum of two periods was allowed for each star. The period search was done independently on the light curve segments from each of the several monitoring runs and then refined using the combined light curves. Stars with periods close to or exceeding the length of one series of observations were analysed only on the basis of the combined light curve. We note that for the typical periods of tens of days found for the variables, the light curves are well sampled so that aliasing should not be a significant problem. Due to the semi-regular nature of the variability in this kind of star, any periodicity found represents only a snapshot of a possibly more complex light curve, and some non-detected periodicities on much longer time scales may exist as well.

\subsection{Near-infrared data}

The ANDICAM instrument offers the possibility to get nearinfrared images at the same time as the optical image. During the time span between October 2003 and January 2004 we obtained 12 epochs of $K$-band data for NGC 2808 and 10 epochs for NGC 362, respectively. However, the field-of-view is significantly smaller in the infrared than in the optical, and the infrared (IR) field is a little bit off center. Therefore only some of the variables identified in the optical could also be measured in the infrared. For NGC 2808 we got time series for 11 stars and a single measurement for two further objects. Only three variables could be observed in NGC 362.

Photometry on the $K$-band images was performed in the same way as for the visual data. The zero point of the magnitude

\footnotetext{
${ }^{1}$ http://stev.oapd.inaf.it/cgi-bin/trilegal
} 


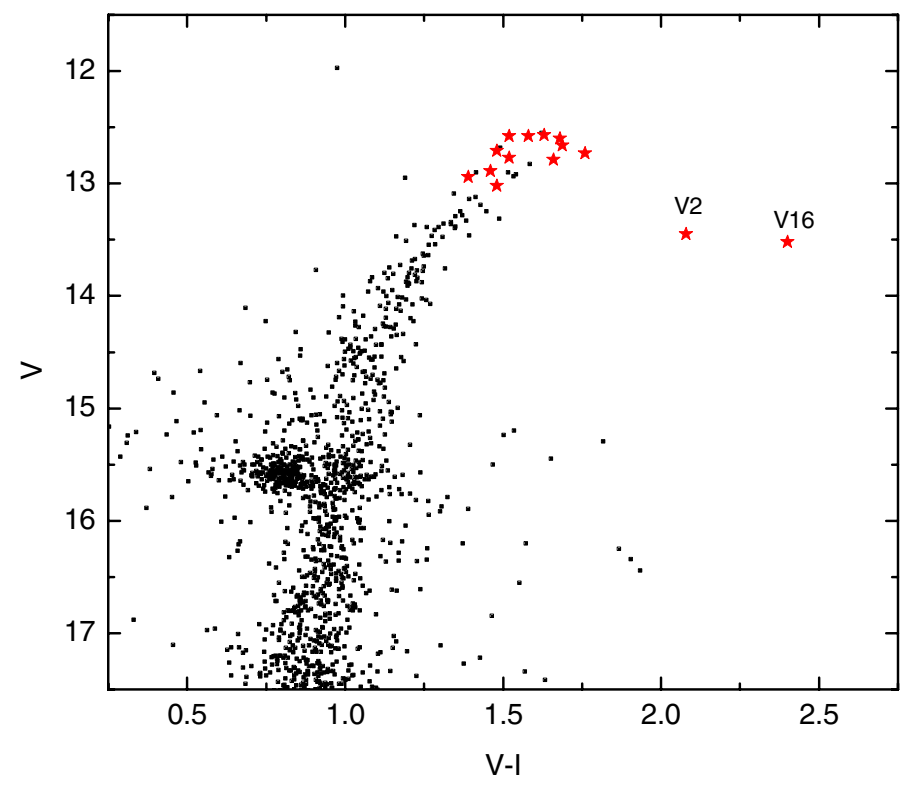

Fig. 1. CMD for NGC 362 derived from ANDICAM data. The long period variables are marked by red stars.

scale was set using 4 to 8 stars on the frame that had 2MASS magnitudes. Observed magnitudes were converted to the 2MASS system by using the relation given in Carpenter (2001). All individual infrared measurements for each variable (detected from visual data) were combined to get an average $K$ magnitude. Using 2 nonvariable 2MASS sources we estimate an accuracy of the mean $K$ brightness of 0.03 mag.

\section{Results}

In both clusters a number of long period variables were found. We identified the variables in the 2 MASS point source catalogue where possible. With the exception of two stars (1 in NGC 362 and 1 in NGC 2808) close to the cluster centre we could in this way attribute coordinates and 2MASS photometry data to our targets.

\subsection{NGC 362}

Four known and seven new long period variables were found in NGC 362. For a large fraction of the variables in this cluster we had to rely on the ANDICAM data only for determining the period, because for the MACHO observations the central part of the cluster unfortunately fell onto damaged areas of the detector for a significant fraction of the time series. The stars with ANDICAM data only are identified in Table 2, where coordinates, $V, I_{\mathrm{C}}, 2 \mathrm{MASS}$ magnitudes and periods are listed. As in Lebzelter \& Wood (2005) we used the variable star identifiers given by Clement et al. (2001) where available. All other variables were named "LW" with some number. Most of these variables have been detected for the first time, see below for a few exceptions. The location of the variables in a CMD of the cluster can be seen in Fig. 1. The phased light curves are presented in Fig. 2.

It is quite obvious that most stars show some kind of irregular behaviour on top of the adopted periodic light change. For some targets a reasonable fit was only possible by adding a long secondary period like in the case of LW8. However, an accurate period for these long time variations could not be determined due to the limited length of our time series. In addition to the variables listed here we noticed three other red stars that showed some kind of light variability, but the variations did not follow any (semi-)periodicity. These stars are listed at the bottom of Table 2, but were not investigated further.

Periods exist in the literature for two stars of our NGC 362 sample. As can be seen we confirm the period of $105 \mathrm{~d}$ for V2 originally found by Sawyer (1931). It is not clear, if the star is changing period from time to time or if the $90 \mathrm{~d}$ period of Eggen (1972) resulted from a short time irregularity in the light curve ${ }^{2}$. The literature period for V16 is also found in our data.

Our star LW1 is identical with the star V36 of Szekely et al. (2007). We confirm the suspicion that the star is a long period variable. A second star from the list of Szekely et al., V56, is located very close to our variable LW6. However, these authors classified it as an eclipsing binary. While we cannot completely reject this hypothesis, we think that a classification as LPV seems to be more probable.

Infrared data were added to Table 2 from the 2MASS catalogue. As mentioned above our ANDICAM near-IR photometry provided multiple $K$-magnitudes for only three sources from our list (LW7, LW8 and LW9). LW7 has a mean $K=8.72 \pm$ $0.05 \mathrm{mag}$, for LW8 we find $K=9.11 \pm 0.05 \mathrm{mag}$. In both cases the scatter is above the expected photometric accuracy, but no reasonable light curve could be extracted. For LW9 we derived an average $K$ magnitude of $8.68 \mathrm{mag}$. The star shows light variations with a $K$ amplitude of up to $0.1 \mathrm{mag}$, which are rather well synchronized with the observed change in the visual. Our $K$-band photometry is in good agreement with the 2MASS data.

Two investigations dedicated to cluster membership of stars in NGC 362 are found in the literature, namely the proper motion study by Tucholke (1992) and the radial velocity study of Fischer et al. (1993). Proper motion data are also found in the NOMAD database (Zacharias et al. 2005). Five stars from our sample have been measured by Tucholke: V2, LW8 and LW11 are listed as proper motion members of the cluster. LW1 and LW9 have a low probability for membership, but the proper motion data for these two stars come with a large error bar. LW8, LW10 and LW11 through LW13 are cluster members according to their radial velocity. The colour-magnitude diagram of the cluster (Fig. 1) together with the above mentioned test using the TRILEGAL stellar population model suggest that all variables from our list are cluster members. While the cluster is seen in projection on the outskirts of the Small Magellanic Cloud (SMC), any contamination of the observed upper giant branch by extragalactic stars is expected to be negligible due to the very low number density of SMC stars in that colour-magnitude range (cf. Udalski et al. 2008).

\subsection{NGC 2808}

Seventeen red variables of NGC 2808 showed a (semi-)periodic light variation, including two variables known before (V1 and V11). Figure 3 shows the location of the variables in a colourmagnitude diagram of the cluster. For 4 stars, all having a comparably short period, only the ANDICAM data, which show a higher sampling in time, were used for the analysis. The corresponding phased light curves using the period with the largest amplitude are shown in Fig. 4. In three cases (LW6, LW9 and LW14) we subtracted a long time trend before producing the

\footnotetext{
2 The time series used by Eggen (1972) to derive the $89 \mathrm{~d}$ period covered only one pulsation cycle.
} 
T. Lebzelter and P. R. Wood: Long period variables and mass loss in the globular clusters NGC 362 and NGC 2808
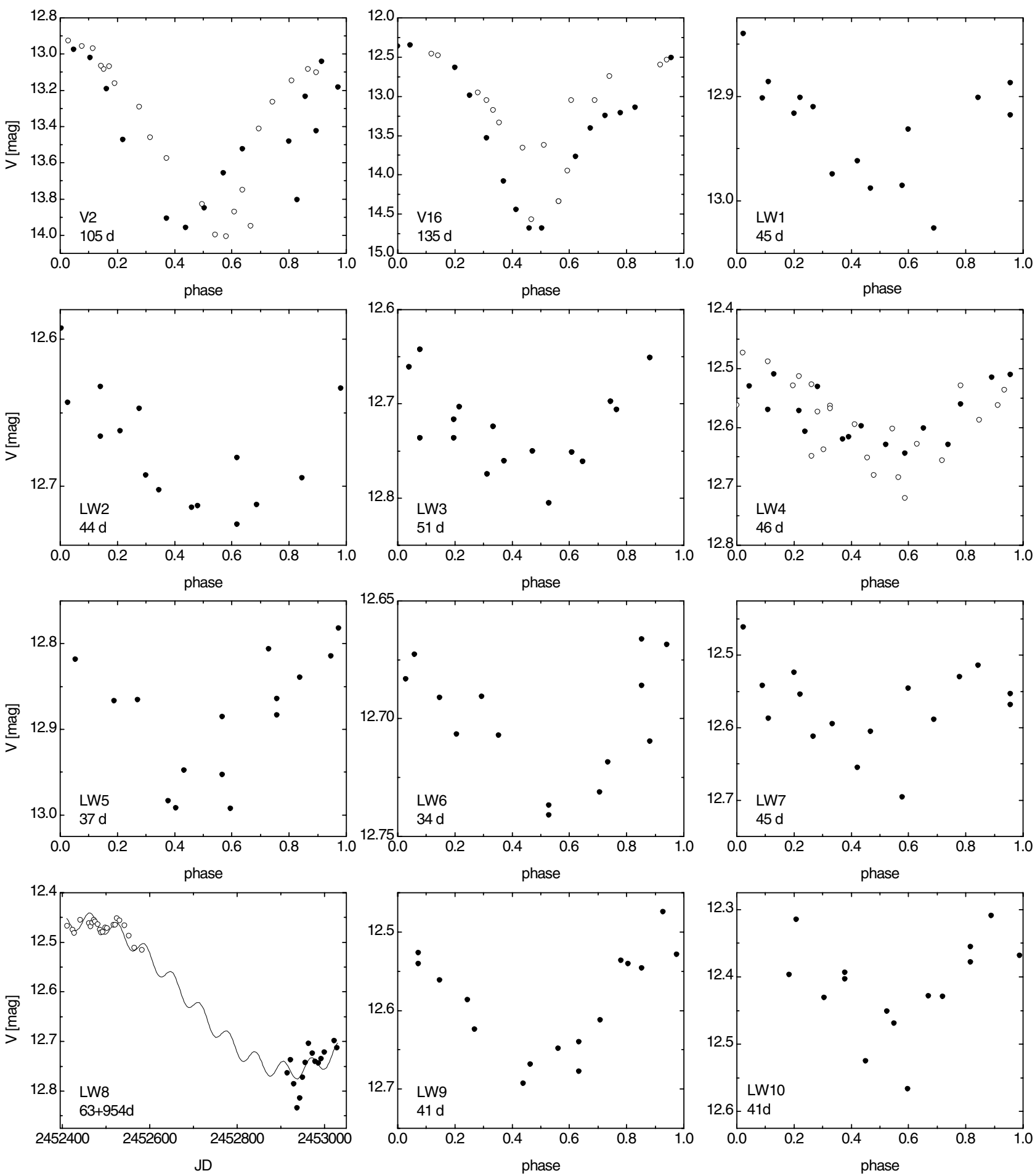

Fig. 2. Phase diagrams of long period variables in our survey of NGC 362. In the lower left corner we show the light change of LW8 against time to illustrate the long-term variation of this star. The MACHO observations are shown as open circles while the ANDICAM observations are shown as filled circles.

phase diagram. For LW2, ANDICAM and MACHO data were analysed independently and both gave a very similar period. In the plot we show only one set of ANDICAM data as there seems to be a phase shift between the older and the more recent time series. In addition to the phase plots shown in Fig. 4, light curves plotted against time are shown in Fig. 5 for selected stars, including those with long term trends, multiple periods or long secondary periods. Beside the stars shown, we found
5 targets located on the upper part of the giant branch, which clearly show variability but without a clearly expressed periodicity. These stars are listed at the bottom of Table 3 .

As with the LPVs in NGC 362, irregularities in the light change can be seen on top of the periodic variations. Some stars, e.g. LW5, show clear signatures of an additional short time variation on top of the longer period. All reliably determined periods are listed in Table 3. There is no source corresponding to LW15 
Table 2. Long period variables of NGC 362.

\begin{tabular}{|c|c|c|c|c|c|c|c|c|c|}
\hline ID & RA (2000) & $\overline{\text { Dec (2000) }}$ & $\begin{array}{c}V^{a} \\
{[\mathrm{mag}]}\end{array}$ & $\begin{array}{c}I_{\mathrm{C}}^{a} \\
{[\mathrm{mag}]}\end{array}$ & $\begin{array}{c}J \\
{[\mathrm{mag}]}\end{array}$ & $\begin{array}{c}H \\
{[\mathrm{mag}]}\end{array}$ & $\begin{array}{c}K \\
{[\mathrm{mag}]}\end{array}$ & $\begin{array}{l}\text { Period } \\
\text { [d] }\end{array}$ & Remark \\
\hline$\overline{\mathrm{V} 2}$ & 010321.9 & -705420.1 & 13.45 & 11.37 & 9.67 & 8.99 & 8.76 & 105 & literature period 90 or $105 \mathrm{~d}$ \\
\hline V16 & 010315.0 & -705032.3 & 13.52 & 11.12 & 9.10 & 8.44 & 8.17 & 135 & literature period $135 \mathrm{~d}$ \\
\hline LW1 & 010307.8 & -704946.5 & 12.94 & 11.55 & 10.32 & 9.60 & 9.47 & 45 & $\begin{array}{c}\text { only ANDICAM data, } \\
\text { V36 of Szekely et al. (2007) }\end{array}$ \\
\hline LW2 & 010310.7 & -705054.0 & 12.66 & 10.97 & 9.85 & 8.97 & 8.75 & 44 & only ANDICAM data \\
\hline LW3 & 010313.6 & -705037.0 & 12.73 & 10.97 & 9.73 & 8.89 & 8.61: & 51 & period from ANDICAM data \\
\hline LW4 & 0103 13.6: & -70 50 13.8: & 12.60 & 10.92 & & & & 46 & no 2MASS identification \\
\hline LW5 & 010315.2 & -70 5103.3 & 12.89 & 11.43 & 10.07 & 8.36 & 9.28: & 37 & only ANDICAM data \\
\hline LW6 & 010317.2 & -705049.7 & 12.71 & 11.23 & 9.41 & 8.70 & 8.47 & 34 & $\begin{array}{l}\text { only ANDICAM data, } \\
\text { probably V56 of Szekely et al. }\end{array}$ \\
\hline LW7 & 010319.0 & -705051.4 & 12.58 & 11.06 & 9.79 & 8.93 & 8.75 & 45 & only ANDICAM data \\
\hline LW8 & 010333.0 & -704937.2 & 12.77 & 11.25 & 10.06 & 9.30 & 9.10 & 63: & long time light trend \\
\hline LW9 & 010335.7 & -705052.1 & 12.58 & 11.00 & 9.74 & 8.91 & 8.70 & 41 & only ANDICAM data \\
\hline LW10 & 010314.5 & -705058.1 & 12.44: & 11.35: & 8.98: & 7.59 & 7.57: & 41 & only ANDICAM data \\
\hline LW11 & 010258.2 & -704944.4 & 13.02 & 11.54 & 10.29 & 9.55 & 9.38 & $\ldots$ & no period found \\
\hline LW12 & 010313.8 & -70 5109.3 & 12.79 & 11.13 & 9.78: & 8.55 & 8.86 & $\ldots$ & no period found \\
\hline LW13 & 010320.1 & -705055.0 & 12.57 & 10.94 & 9.72 & 8.94 & 8.72 & $\ldots$ & no period found \\
\hline
\end{tabular}

Notes. A colon indicates a number with more uncertainty than usual. ${ }^{(a)}$ Mean values.

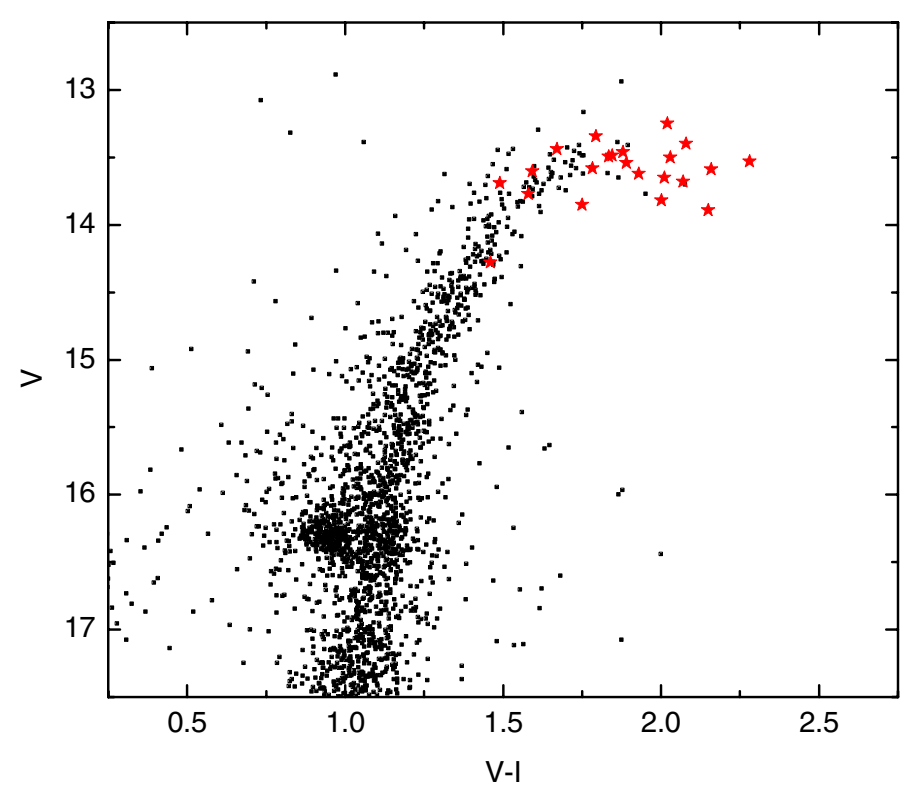

Fig. 3. CMD for NGC 2808 derived from ANDICAM data. The long period variables are marked by red stars.

in the 2MASS catalogue. For this star the coordinates are estimated using the SIMBAD/Aladin tool on the 2MASS $J$-band image.

We also list in Table 3 our own $K$-band photometry (transfered to the 2MASS system) where available. The given uncertainty of the $K$-band data includes both the measurement error and the star's near-infrared variability during the four months of monitoring. For V11, LW9, LW18 and LW19 we have only a single epoch measurement. A well expressed near-infrared variability was detected for three stars in our sample, V1, LW1 and LW15. The light curves are shown in Fig. 6 together with the visual data obtained at the same time. It can be seen that for LW1 and LW15 the optical and infrared lightcurves are nicely in phase, while for V1 there is a large difference. The reason for that is not clear. The slightly larger uncertainty of LW6 is most likely due to a higher photometric error rather than due to variability. The star has another near-infrared source nearby so that blending becomes a problem. Note that for LW15 we see the $332 \mathrm{~d}$ period only as a general trend of the average brightness in Fig. 6. The star obviously shows also a short period change of about 50 days length, which can be seen as some scatter in Fig. 4.

For most sources we find a good agreement of our $K$-band measurement with the 2MASS value taking into account the variability of the objects. One exception is V1. Looking at the near infrared light curve of V1 (Fig. 6) the 2MASS value would suggest an amplitude and thus a period much longer than the one measured from the visual (the plotted range covers almost one complete light cycle). Therefore we may speculate that the infrared light curve maybe expresses some long time variation. There is no indication for that in the visual light curve. For two further stars, LW7 and LW19, the difference between our value and the 2MASS data is also clearly exceeding the typical uncertainty or variability scatter. We think the reason for this is an error in the 2MASS value. For both stars the data point in the 2MASS catalogue received a low quality flag, probably due to crowding. For LW7, our value is the average of 11 measurements at different epochs giving a standard deviation of $\sigma=0.07 \mathrm{mag}$. The higher value for $\sigma$ compared to some of the other low amplitude variables in Table 3 may be understood by the fact that it is the faintest star in our sample. For LW19 we have only a single measurement.

No proper motion study for individual stars in NGC 2808 could be found in the literature. Membership information for our variables comes from the study of red giant stars in this cluster by Cacciari et al. (2004). They find a mean radial velocity of approximately $101 \pm 10 \mathrm{~km} \mathrm{~s}^{-1}$ for a sample of $132 \mathrm{ob}$ jects they consider cluster members. They also note that they found 5 additional objects with "strongly discrepant" velocities, and they presumed these to be field stars. The variables V1, LW1, LW3, LW5, LW6, LW9, LW10, LW12-14 and LW17 have a measured radial velocity in Cacciari et al. (2004). In all cases, there is a strong indication that these variables are 
T. Lebzelter and P. R. Wood: Long period variables and mass loss in the globular clusters NGC 362 and NGC 2808
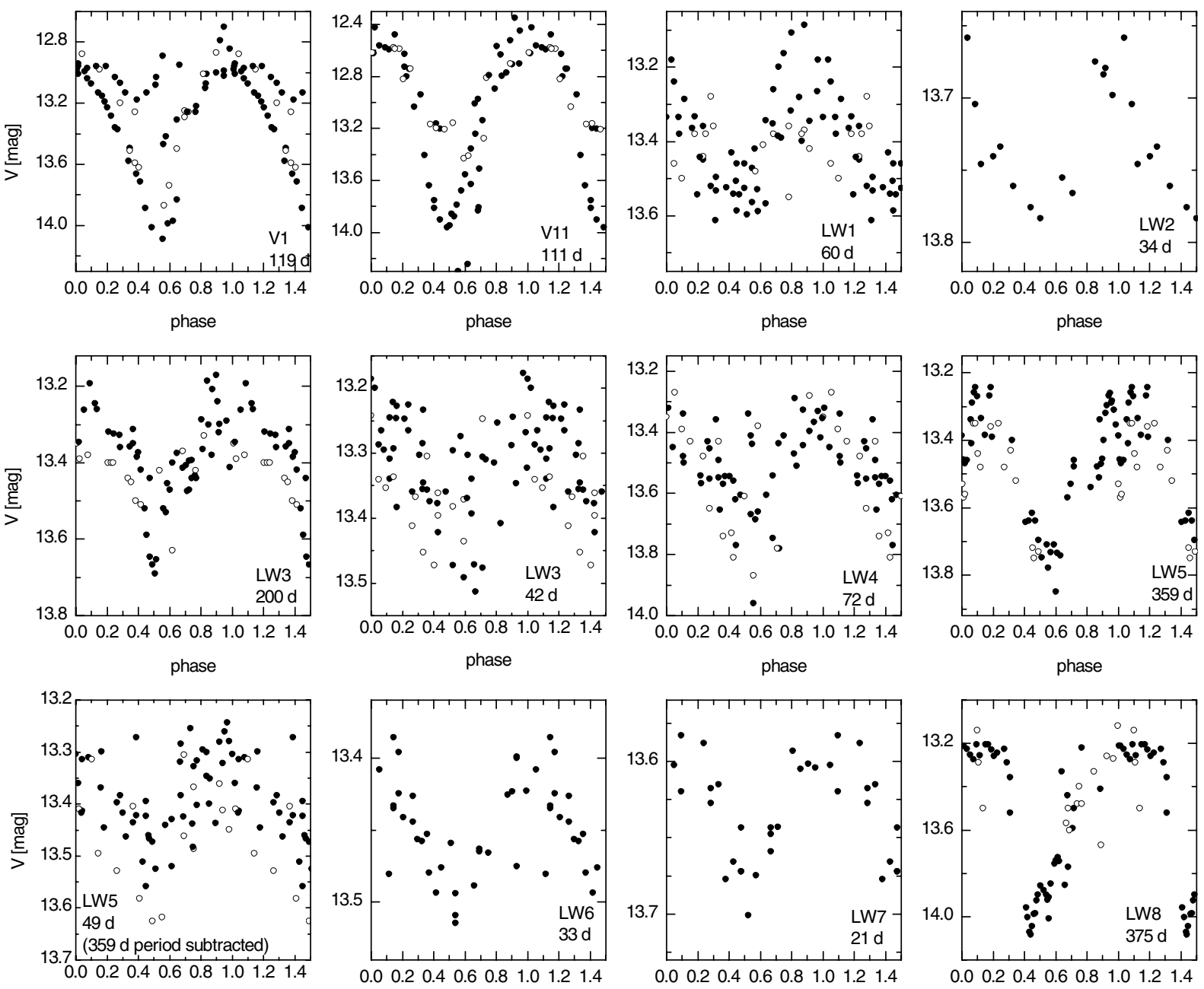

phase
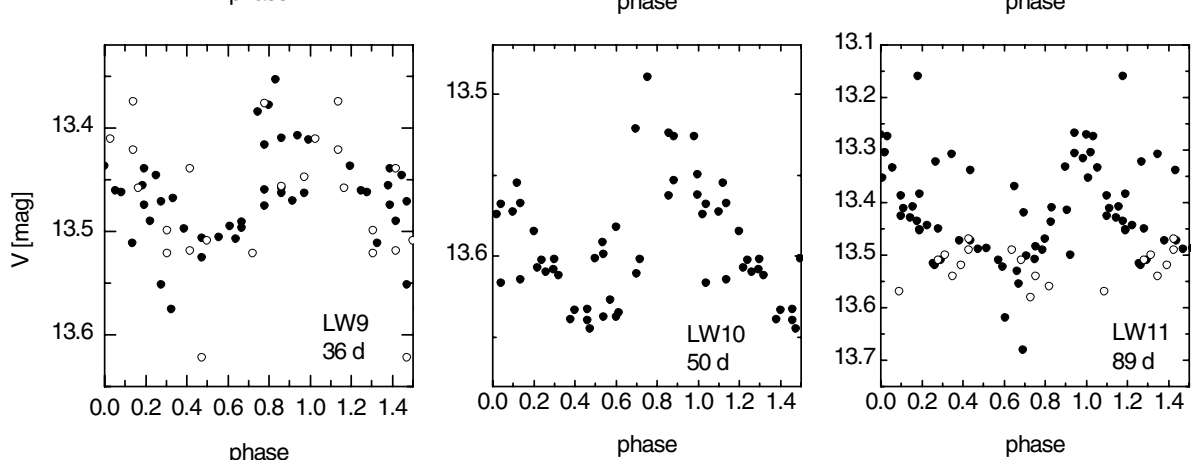

phase
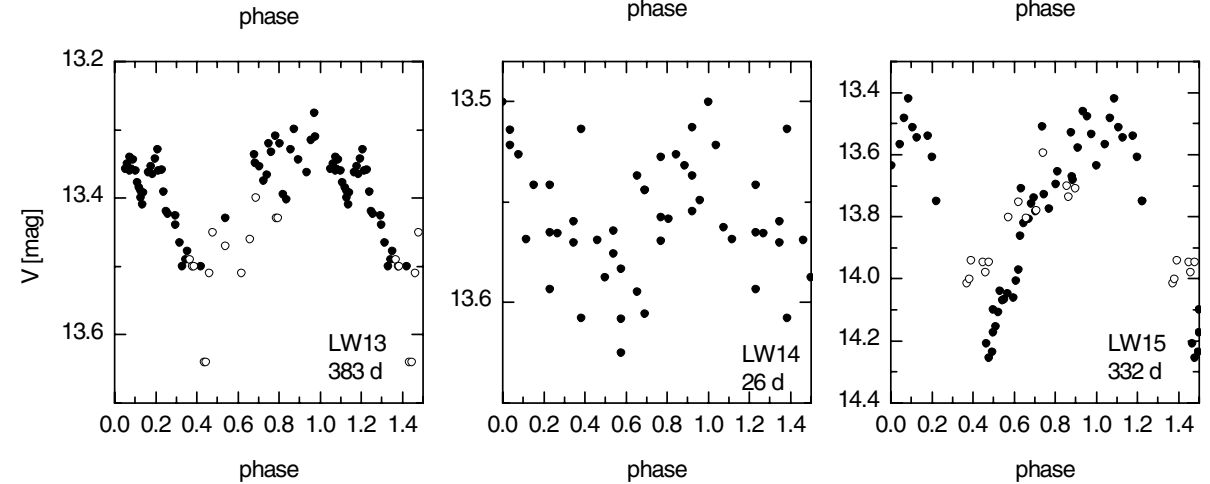

Fig. 4. Phase diagrams of long period variables in our survey of NGC 2808. The MACHO observations are shown as open symbols while the ANDICAM observations are shown as filled symbols. 
Table 3. Long period variables of NGC 2808.

\begin{tabular}{|c|c|c|c|c|c|c|c|c|c|c|}
\hline ID & $\overline{\text { RA (2000) }}$ & $\overline{\operatorname{Dec}(2000)}$ & $\begin{array}{c}V^{a} \\
{[\mathrm{mag}]}\end{array}$ & $\begin{array}{c}I_{\mathrm{C}}{ }^{a} \\
{[\mathrm{mag}]}\end{array}$ & $\begin{array}{c}J \\
{[\mathrm{mag}]}\end{array}$ & $\begin{array}{c}H \\
{[\mathrm{mag}]}\end{array}$ & $\begin{array}{c}K \\
{[\mathrm{mag}]}\end{array}$ & $\begin{array}{c}K_{\text {andi }} \\
{[\mathrm{mag}]}\end{array}$ & $\begin{array}{l}\text { Period } \\
\text { [d] }\end{array}$ & Remark \\
\hline$\overline{\mathrm{V} 1}$ & 091220.2 & -645220.5 & 13.53 & 11.25 & 10.27 & 9.50 & 9.16 & $8.60 \pm 0.06$ & 119 & \\
\hline V11 & 091206.7 & -645240.3 & 13.25 & 11.23 & 9.77 & 9.04 & 8.82 & $8.92^{b}$ & 111 & \\
\hline LW1 & 091157.1 & -64 5129.7 & 13.40 & 11.32 & 9.95 & 9.10 & 8.83 & $8.77 \pm 0.03$ & 60 & \\
\hline LW2 & 091158.7 & -645128.5 & 13.77 & 12.19 & 10.92 & 10.17 & 9.95 & $9.92 \pm 0.03$ & 34 & ANDICAM only \\
\hline LW3 & 091159.2 & -645259.2 & 13.50 & 11.47 & 10.02 & 9.07 & 8.85 & $8.83 \pm 0.03$ & 42,200 & 2 periods \\
\hline LW4 & 091201.4 & -645137.4 & 13.65 & 11.64 & 10.24: & 9.06: & 9.24: & $9.16 \pm 0.07$ & 72 & \\
\hline LW5 & 091201.7 & -645033.2 & 13.59 & 11.43 & 10.18 & 9.26 & 9.02 & & 49,359 & 2 periods \\
\hline LW6 & 091202.3 & $-6451 \quad 18.4$ & 13.49 & 11.64 & 10.34 & 9.49 & 9.24 & $9.16 \pm 0.07$ & 33 & long time trend \\
\hline LW7 & 091202.7 & -645201.7 & 13.69 & 12.20 & 10.68: & 10.03: & 9.80: & $10.14 \pm 0.07$ & 21 & ANDICAM only \\
\hline LW8 & 09 12 04.3: & -64 51 41.7: & 13.68: & 11.61: & 9.86: & 8.96: & 8.75: & $8.87 \pm 0.12$ & 375: & \\
\hline LW9 & 091206.7 & -645218.1 & 13.54 & 11.65 & 10.23 & 9.35 & 9.10 & $9.08^{b}$ & 36 & ANDICAM only \\
\hline LW10 & 091208.5 & -645158.4 & 13.62 & 11.69 & 10.34 & 9.43 & 9.25 & & 50 & long secondary $\mathrm{P}$ \\
\hline LW11 & 091211.3 & -645242.2 & 13.52 & 11.66 & 10.29 & 9.35 & 9.17 & $9.07 \pm 0.05$ & 89: & \\
\hline LW12 & 091214.5 & -645326.7 & 13.34 & 11.55 & 10.18 & 9.30 & 9.07 & & 42 & ANDICAM only \\
\hline LW13 & 091216.6 & -645202.8 & 13.46 & 11.58 & 10.23 & 9.35 & 9.10 & $9.05 \pm 0.05$ & 383 & prob. also short $\mathrm{P}$ \\
\hline LW14 & 091218.5 & -645130.6 & 13.60 & 12.01 & 10.87 & 10.10 & 9.91 & $9.85 \pm 0.05$ & 26 & ANDICAM only \\
\hline LW15 & (09 12 (203.9) & $(-645154.2)$ & 13.89: & 11.74: & & & & $8.93 \pm 0.09$ & 332 & $\begin{array}{l}\text { no } 2 \text { MASS ID } \\
\text { (crowded) }\end{array}$ \\
\hline LW16 & 091150.3 & -645142.2 & 13.82 & 11.82 & 10.24 & 9.33 & 9.10 & $9.13 \pm 0.03$ & $\ldots$ & no period found \\
\hline LW17 & 091201.5 & -645049.3 & 13.85 & 12.10 & 10.90: & 9.25: & 9.95: & & $\ldots$ & no period found \\
\hline LW18 & 091204.9 & -645129.7 & 14.28 & 12.82 & 11.57 & 11.38 & 10.77 & $10.81^{b}$ & $\ldots$ & no period found \\
\hline LW19 & 091205.9 & -645208.1 & 13.58 & 11.80 & 10.38: & 9.51: & 9.24: & $9.39^{b}$ & $\ldots$ & no period found \\
\hline LW20 & 091224.8 & $-6451 \quad 10.3$ & 13.44 & 11.77 & 10.38 & 9.49 & 9.29 & & $\ldots$ & no period found \\
\hline
\end{tabular}

Notes. A colon indicates a number with more uncertainty than usual. ${ }^{(a)}$ Mean values. ${ }^{(b)}$ Only one single measurement in the $K$-band.
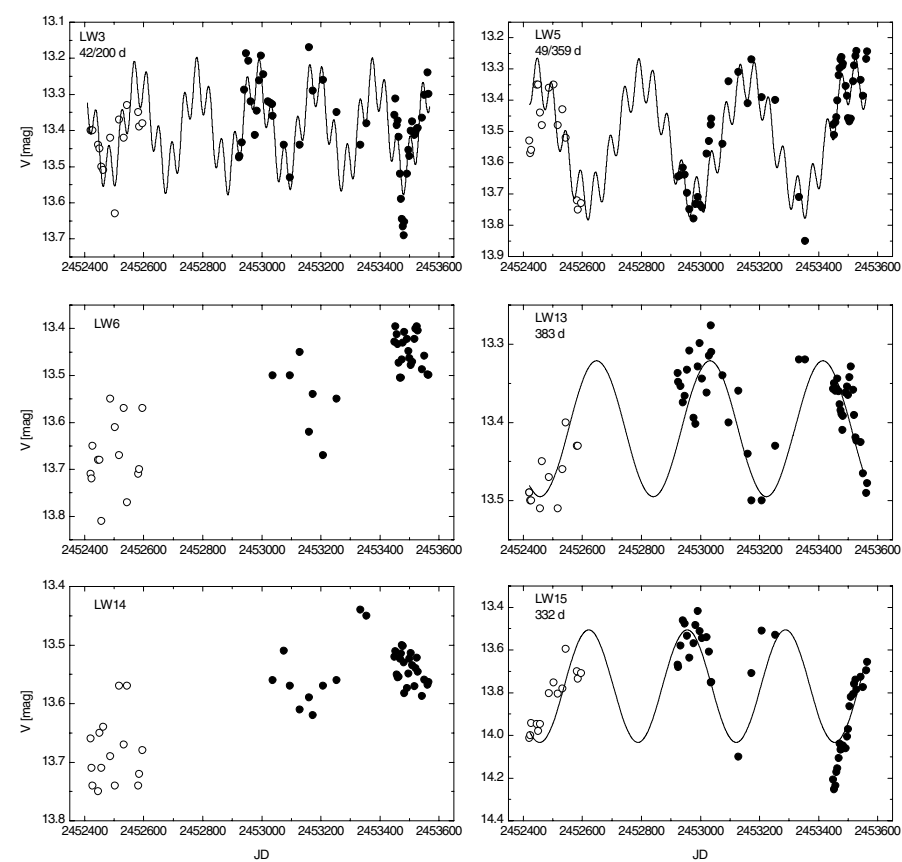

Fig. 5. Photometry of several variables in NGC 2808 plotted against Julian Date. The corresponding Fourier fits are shown for stars with long periods. For the two stars with long term trends in magnitude, only the observations are shown. The MACHO observations are shown as open symbols while the ANDICAM observations are shown as filled symbols.

cluster members, with the variables having a mean radial velocity of $100.8 \pm 11.6 \mathrm{~km} \mathrm{~s}^{-1}$. There were no stars with strongly discrepant velocities. Statistically, the results of Cacciari et al. (2004) suggest that the field population is about 3.6\% (5 out of
137 objects) which would indicate that there could be about 0.8 field stars in our sample of 22 variables in NGC 2808. As in the case of NGC 362 the CMD (Fig. 3) also suggests that all the variables belong to this cluster.

\section{The $\log P-K$ and $\log P-M_{\mathrm{bol}}$ diagrams}

As can be seen from the CMDs of the two clusters (Figs. 1 and 3) all our variables are indeed long period variables on the uppermost part of the giant branch and thus either AGB stars or stars near the tip of the first giant branch. Furthermore we may notice that there is no non-variable star at the tip of the giant branch. The same result was also found in 47 Tuc (Lebzelter \& Wood 2005).

Using these variables we constructed a $\log P-K$ diagram. Due to the similarity in metallicity and age of the two clusters we decided to combine the data into one diagram, but use separate symbols for the two clusters. Only stars with a well defined periodicity and $K$ brightness were taken into account (no colon after the period or $K$ magnitude in Tables 2 or 3 ). The observed apparent $K$ magnitudes were corrected for reddening, using $E(B-V)=$ $0.05 \mathrm{mag}$ for NGC 362 and $E(B-V)=0.23 \mathrm{mag}$ for NGC 2808, and distance, using $(m-M)_{V}=14.80$ and $15.56 \mathrm{mag}$, respectively (we have taken these numbers from Piotto et al. 2002). The reddening law adopted (Rieke \& Lebofsky 1985) gives $A_{V}=3.1 E(B-V)$ and $A_{K}=0.35 E(B-V)$. In NGC 2808, we used the ANDICAM $K$ magnitude in preference to the 2MASS value.

In Fig. 7 we present the combined $\log P-K$ diagram together with the LMC relations given by Ita et al. (2004). The variables found in 47 Tuc are also shown for comparison. NGC 362 and NGC 2808 indeed seem to occupy a very similar area in the $\log P-K$ diagram supporting our approach to combine the two data sets in our analysis. For an interpretation of this plot let us 
T. Lebzelter and P. R. Wood: Long period variables and mass loss in the globular clusters NGC 362 and NGC 2808

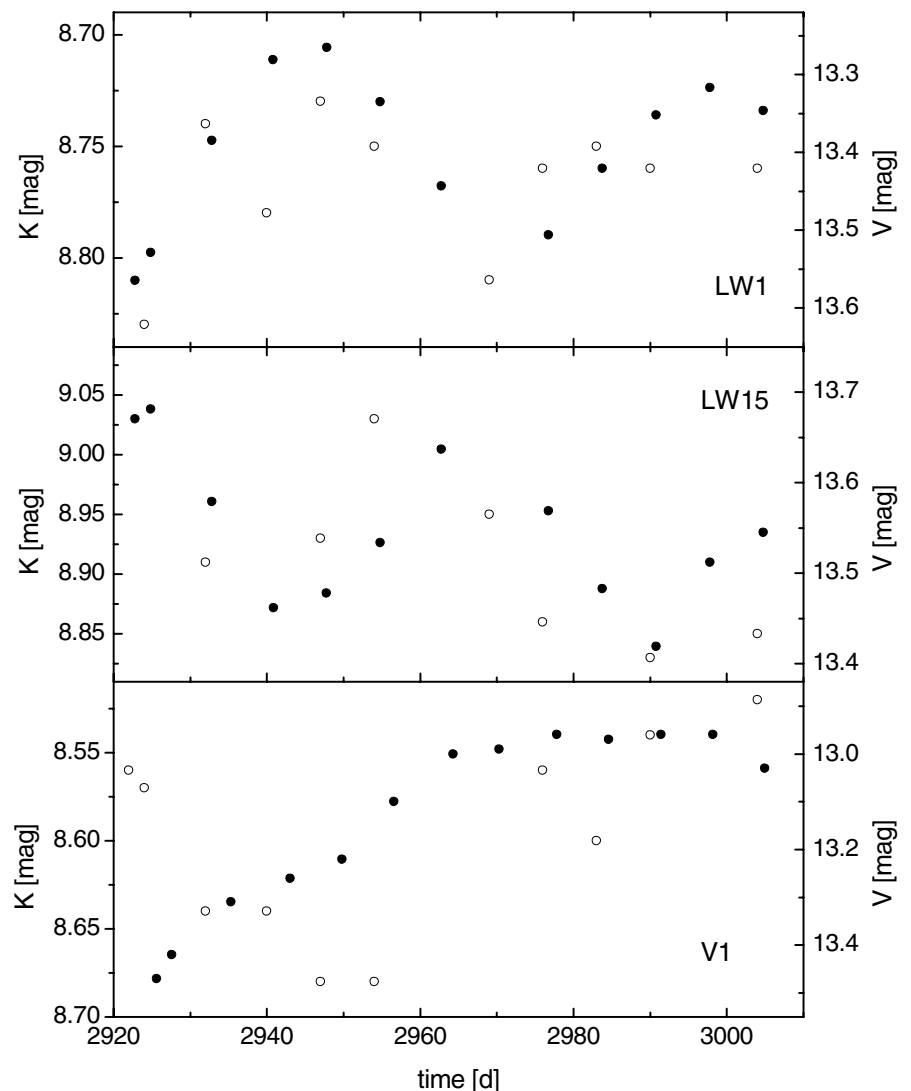

Fig. 6. Near infrared light curve (solid symbols, left $y$-axis) and visual light curve (open symbols, right $y$-axis) for LW1, LW15 and V1 of NGC 2808 (from top to bottom). Near infrared and visual data have been obtained at the same time.

recall the differences in the global parameters of NGC 362 and NGC 2808, 47 Tuc and the LMC. 47 Tuc is more metal rich than NGC 362 and NGC 2808. As summarized in the introduction section of this paper and in Lebzelter \& Wood (2005), different relative ages have been found for these three clusters and consequently they will have somewhat different mass stars on the AGB. The average metallicity of the LMC (from which Ita et al. 2004 , derived their $\log P-K$ relations) is higher than any of the clusters. As the LMC is certainly not a single age stellar population, the LPVs found are expected to represent a wide range of stellar masses. It is not therefore clear that the Ita et al. sequences should give a close fit to the NGC 362 and NGC 2808 data.

There is a clear difference both in the maximum and in the average brightness of the LPVs in NGC 362 and NGC 2808 compared with 47 Tuc. The most luminous star (in the $K$-band) in 47 Tuc is almost 0.8 mag brighter than the brightest LPV in NGC 362. Similarly, the lowest luminosity LPVs found in 47 Tuc are brighter than the lowest luminosity LPVs found in NGC 362 and NGC 2808. Part of the reason for the offset in $K$ is that the 47 Tuc stars are slightly cooler and their bolometric corrections are larger than those of the NGC 362 and NGC 2808 stars. However, even in the $\log P-M_{\text {bol }}$ diagram (Fig. 8), there still remains an offset of $\sim 0.5 \mathrm{mag}$. This difference is larger than the difference in RGB tip luminosity which is $\sim 0.15$ mag brighter in 47 Tuc than in NGC 362 and NGC 2808 according to the evolutionary tracks of Bertelli et al. (2008) for $Z=0.004$ and $Z=0.001$, respectively.

The same selection criteria were applied on all cluster data sets, and the image quality was very similar so that variables

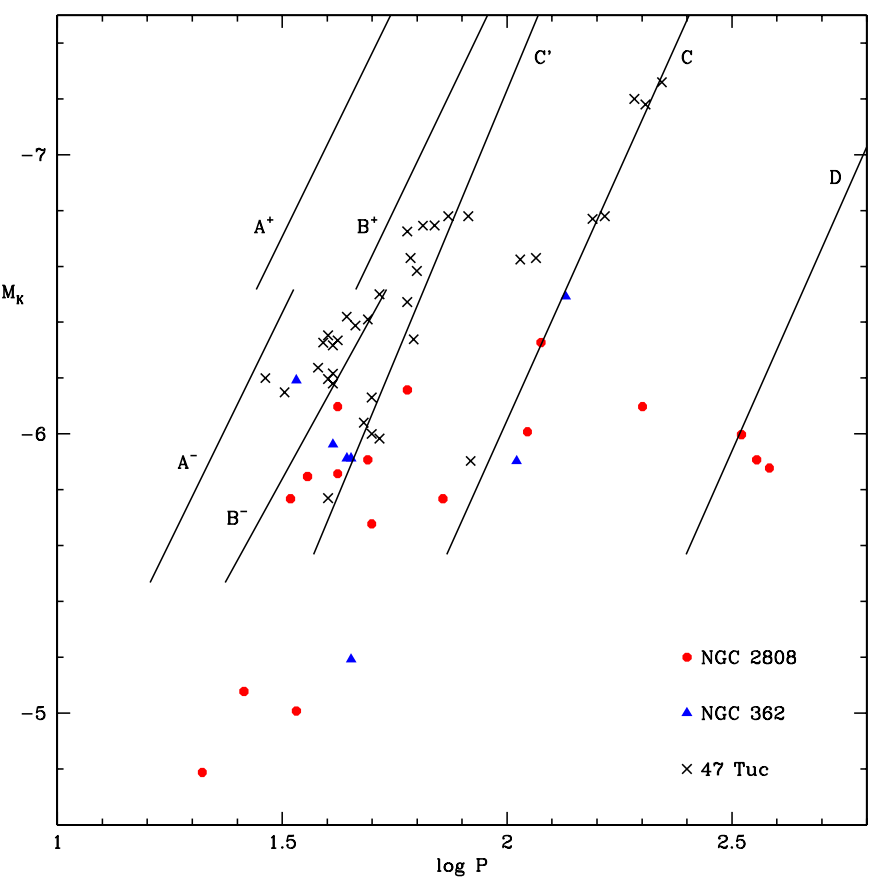

Fig. 7. LPVs in 47 Tuc, NGC 362 and NGC 2808 plotted in the $\log P, M_{K}$ diagram along with the fits to the various LMC sequences given by Ita et al. (2004).

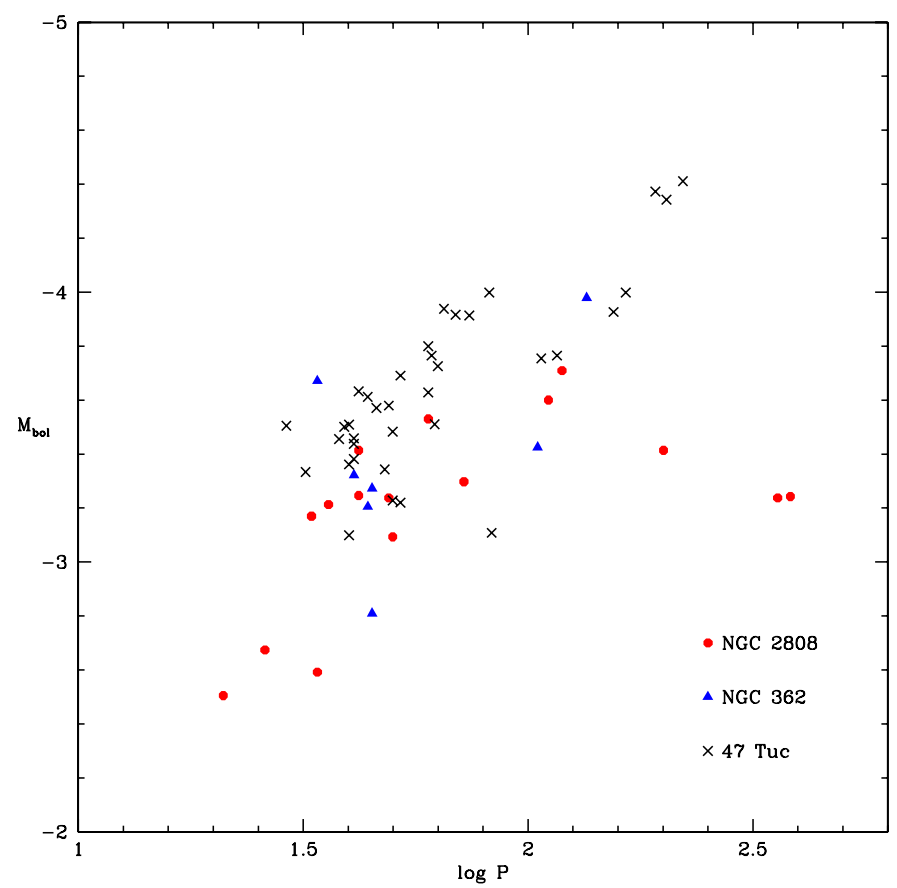

Fig. 8. The $\log P, M_{\mathrm{bol}}$ diagram for stars in 47 Tuc, NGC 362 and NGC 2808.

should have been detected to similar magnitude limits. We therefore reach the conclusion that the LPV distribution in NGC 362 and NGC 2808 is $~ 0.5$ mag fainter than in 47 Tuc.

In general, it is interesting to note that we found considerably fewer LPVs in NGC 362 and NGC 2808 than in 47 Tuc. Large amplitude variables $(\Delta V>2 \mathrm{mag})$ are missing in NGC 362 and NGC 2808. Frogel \& Elias (1988) suspected that there are no 
LPVs below a metallicity of $[\mathrm{Fe} / \mathrm{H}] \approx-1$. However, they defined LPVs on the basis of the light amplitude only. The findings from our study shows that this is not true if we consider low amplitude stars. Note also that fundamental pulsators (sequenceC) are present in both clusters. In this context it is also interesting to mention the detection of two likely LPVs detected in the very metal poor cluster M $15([\mathrm{Fe} / \mathrm{H}]=-2.3)$ by McDonald et al. (2010a). Their variations places them onto the pulsation sequence of the long secondary periods.

In 47 Tuc we saw a clear change of pulsation mode around $M_{K}=-6.8 \mathrm{mag}$. Similarly, in the two clusters studied here, overtone pulsators extend up to a cutoff magnitude of $M_{K} \sim-6.2 \mathrm{mag}$, which is $\sim 0.6 \mathrm{mag}$ fainter than in 47 Tuc. There are two LPVs brighter than this pulsating in the fundamental mode and, unlike in 47 Tuc, there are a few stars below the maximum overtone magnitude that also appear to belong to the fundamental mode sequence.

Figure 7 shows three stars in NGC 2808 that obviously belong to sequence-D. No similar stars from 47 Tuc or NGC 362 are seen in this figure. However, this is largely the result of an inability to determine reliable periods for the LSPs in these clusters. In fact, Lebzelter \& Wood (2005) note that 12 of the red variable in 47 Tuc show a long period but these periods could not be determined from the relatively short duration of the observations. The star LW3 in NGC 2808 which sits by itself at $\log P \sim 2.3$ and $M_{K} \sim-6.1 \mathrm{mag}$ is discussed in Sect. 6 .

\section{Observations of mass loss}

In our previous paper on 47 Tuc (Lebzelter \& Wood 2005), we showed that linear pulsation models including some parameterized mass loss can describe the observed $\log P-K$ relations of single stellar populations much better than models without mass loss. These models were then used to determine the mass loss rate on the giant branch. Before we make an attempt to derive the mass loss on the red giant branches of NGC 362 and NGC 2808 from their $\log P-K$ diagrams, we want to briefly review previous studies that have investigated the mass loss in these two clusters.

In NGC 362 the two previously known variables V2 and V16 were found to show an infrared excess suggesting emission from circumstellar dust (Ita et al. 2007). The most recent study of the dust mass loss from giants in NGC 362, also studying an excess in the mid-infrared range, comes from Boyer et al. (2009). We will not discuss here the numbers they give for the mass loss rate since they depend on several assumptions, but will focus instead on their identification of stars with a strong infrared excess. Some of the sources they found in their work had shown up previously in the investigations of Origlia et al. (2002) and McDonald \& van Loon (2007). Boyer et al. identify 5 candidates for large mass loss rate and 5 candidates for moderate mass loss rate from Spitzer photometry. Among the large mass loss rate candidates they found two variables, namely V2 and V16. Sloan et al. (2010) investigated these two stars using IRS onboard the Spitzer space telescope, but found the mid-IR spectrum featureless. They concluded that the infrared excess is caused by iron grains (cf. McDonald et al. 2010b). We identify another star of the list of Boyer et al., s07, as variable LW3. Their source s05 seems to consist of two stars, one of which is LW6. They note that their fifth source with a large mass loss rate, s08, is possibly a stellar blend. We found no variable star at that position. Thus, except for the uncertain case of s08, our data show that all stars in NGC 362 with a strong mid-infrared excess and deduced large mass loss rate are indeed long period variables. Among the 5 stars with moderate mass loss rate from Boyer et al. (2009) we can also identify 4 as variables. Most interesting is the case of s03 which corresponds to our variable LW10, the star detected from its near-infrared variability. The sources s01, s04 and s09 correspond to LW9, LW7 and LW2, respectively. The fifth star of Boyer et al. is outside the field of our study. This comparison clearly shows the strong correlation between high mass loss rates and variability.

Mauas et al. (2006) derive a mass loss rate of a few $10^{-9} M_{\odot}$ on the upper part of the giant branch of NGC 2808 from the analysis of chromospheric lines. $\mathrm{H} \alpha$ line components at an outflow velocity were found in a large sample of red giants by Cacciari et al. (2004). A Spitzer study of this cluster has been obtained already (Fabbri et al. 2008), but the results are not available yet. From the horizontal branch morphology of this cluster D'Antona $\&$ Caloi (2008) and Dalessandro et al. (2011) derive an average mass lost before the red clump phase of $0.15-0.20 M_{\odot}$.

\section{Pulsation models and mass loss}

We have shown that modelling of the LPVs in 47 Tuc allows one to derive the amount of mass lost on the RGB (Lebzelter \& Wood 2005). It is our aim to carry out the same pulsation analysis for NGC 362 and NGC 2808. In addition, given the large range in the helium abundance suspected in NGC 2808, we aim to see how the helium abundance affects the LPV pulsation periods. In particular, we aim to see if the position on the period-luminosity diagram can give a clue to the helium abundance.

Based on the summary of abundance measurements in the two clusters detailed in the Introduction, we assume a metal abundance $Z=0.001$ for both clusters. Models have been made with helium mass fractions $Y=0.25,0.3$ and 0.4 . Note that these are, respectively, the estimated helium mass fractions given by Dalessandro et al. (2011) for red horizontal branch, blue horizontal branch and extreme horizontal branch stars in NGC 2808. Mass loss was added to some models assuming a Reimers' Law (Reimers 1975). The Reimers mass loss rate was multiplied by a factor $\eta=0.4$. This factor was chosen as it leads to a termination of the AGB near the maximum observed luminosity of the variable stars in NGC 362 and NGC 2808. It also closely reproduces the masses estimated for the horizontal branch stars in the two clusters (see below), and it is close to the value of 0.33 we found to apply in 47 Tuc.

In order to compute the declining stellar mass along the RGB and AGB in the presence of mass loss, evolution rates as a function of luminosity were obtained from the evolution tracks of Bertelli et al. (2008). $T_{\text {eff }}$ in our calculations was forced to be the same as the mean observed $T_{\text {eff }}$ on the giant branch (see Figs. 9 or 11) so that the radius used in the Reimers' Law formula was appropriate for NGC 362 and NGC 2808.

Following the discussion in the Introduction, we have assumed an age of $10 \times 10^{9}$ years for both clusters and for each population of different helium abundance. The tracks and isochrones of Bertelli et al. (2008) were used to obtain the initial mass appropriate for giant branch stars of this age, $Y$ and $Z$. Initial masses of $0.86,0.79$ and $0.65 M_{\odot}$ were found for helium abundances of $0.25,0.3$ and 0.4 , respectively.

Linear pulsation models were computed with the code described in Lebzelter \& Wood (2005). A mixing length of 1.7 pressure scale heights was used in all models as this was found to reproduce the observed $T_{\text {eff }}$ of the giant branch well.

Models without mass loss are shown in the HertzsprungRussell-diagram in Fig. 9. The $M_{\text {bol }}$ and $T_{\text {eff }}$ values of the cluster stars shown in this figure were obtained from $K$ and $J-K$ values from 2MASS for non-variable stars or from Tables 2 and 3 
T. Lebzelter and P. R. Wood: Long period variables and mass loss in the globular clusters NGC 362 and NGC 2808

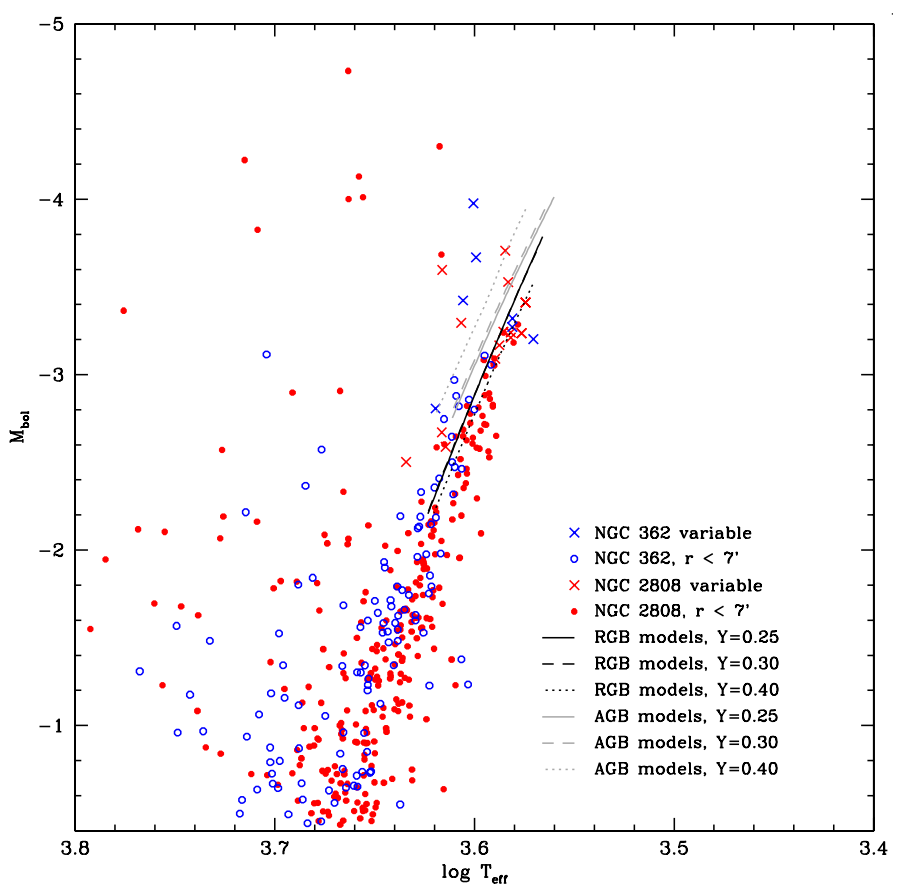

Fig. 9. Combined NGC 362 and NGC 2808 HR-diagram. Luminosity and $T_{\text {eff }}$ were computed from $J$ and $K$ magnitudes (see text). The locus of pulsation models without mass loss is shown for RGB and AGB stars with helium abundance $Y=0.25,0.30$ and 0.40 . All models have metallicity $Z=0.001$.

for variable stars. Bolometric corrections for the $K$ magnitude and the transformation from $J-K$ to $T_{\text {eff }}$ given by Houdashelt et al. $(2000 \mathrm{a}, \mathrm{b})$ were used. The distance moduli and reddenings we used are given in Sect. 4. In the HR-diagram, it can be seen that the models have temperatures in the middle of the range of values of the variables.

The variables are shown in the $\log P-K$ diagram in Fig. 10. For NGC 2808, where a wide range of helium abundance is suspected, a model can be found to fit most variables. However, in the main group of stars brighter than $M_{K}=-5.5$, nearly all the fundamental mode pulsators would require $Y \sim 0.4$, which seems unlikely. Also, the fundamental mode pulsator with $\log P \sim 2.3$ has no explanation. Since its amplitude is low, it is unlikely that the period has changed as a result of large amplitude pulsation, an effect demonstrated by Lebzelter \& Wood (2005) for the large amplitude Mira variables in 47 Tuc. Finally, we note that the 3 stars with $\log P \sim 2.5$ belong on sequence-D as shown in Fig. 7. The origin of their variability is unknown (e.g. Wood et al. 2004; Nicholls et al. 2009).

For NGC 362, where $Y=0.25$ should be appropriate, the models without mass loss are a very poor fit to the observed $\log P, K$ values of the variables. We show below that a significant amount of mass loss on the RGB can explain the periods of the LPVs in NGC 362 and NGC 2808.

Models with mass loss are shown in the HertzsprungRussell-diagram in Fig. 11. With the adopted scaling parameter $\eta=0.4$, it was found that the mass at the tip of the RGB (and hence on the horizontal branch) was $0.693,0.623$ and $0.486 M_{\odot}$ for $Y=0.25,0.30$ and 0.40 , respectively. This is in good agreement with the values of $0.69,0.565$ and $0.479 M_{\odot}$ found by Dalessandro et al. (2011) for red, blue, and extreme horizontal branch stars in NGC 2808. Note that the AGB stars with $Y=0.30$ lose all their H-rich envelope at $M_{\mathrm{bol}} \sim-3.7 \mathrm{mag}$

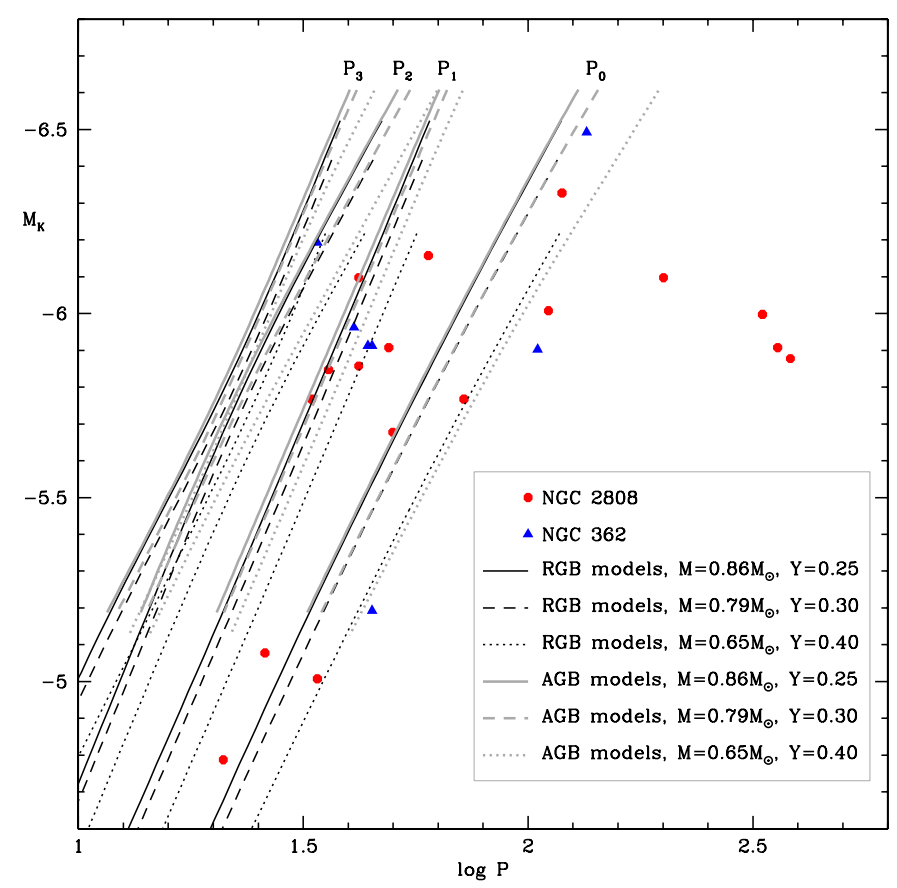

Fig. 10. The $\log P-K$ diagram for cluster variables and models without mass loss. The labels $P_{0}, P_{1}, P_{2}$ and $P_{3}$ indicate radial pulsation in the fundamental mode and the first, second and third overtone modes, respectively.

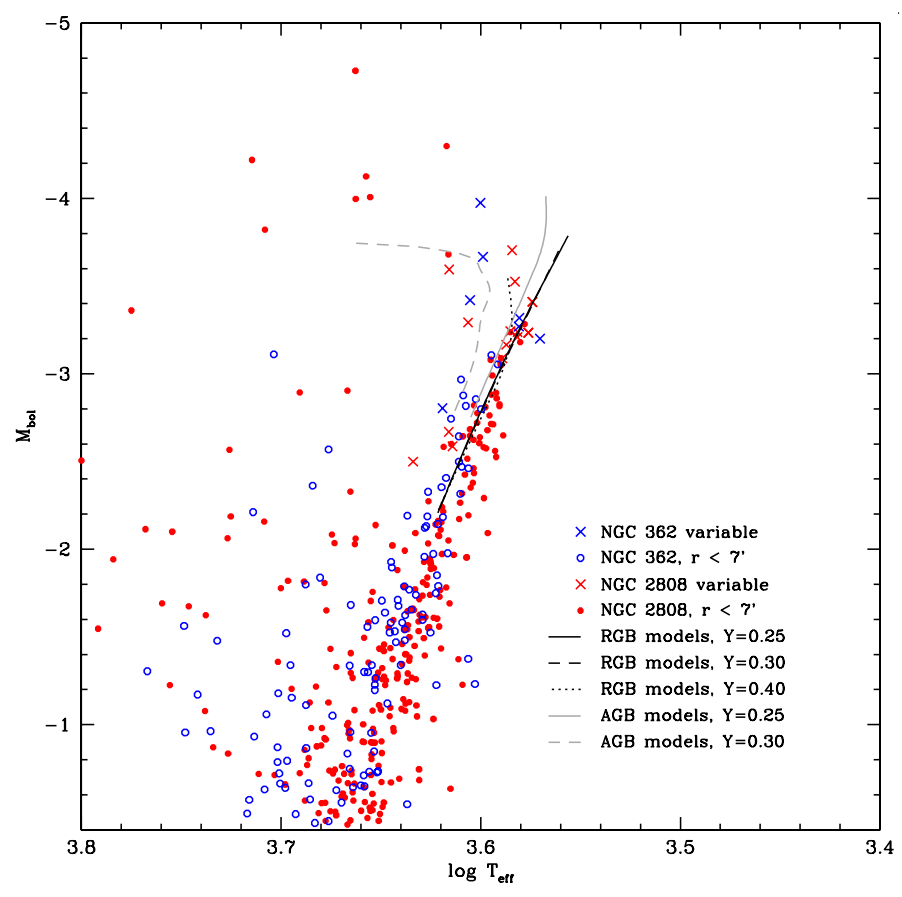

Fig. 11. Same as Fig. 9 but for models with mass loss.

and then evolve away from the AGB to the blue. Even more extreme are the stars with $Y=0.4$ which do not evolve up the AGB at all since their H-burning shells consume the remaining envelope hydrogen while on the horizontal branch. In general, the $M_{\text {bol }}$ and $T_{\text {eff }}$ values of models fall in the middle of the values obtained for the cluster stars. 


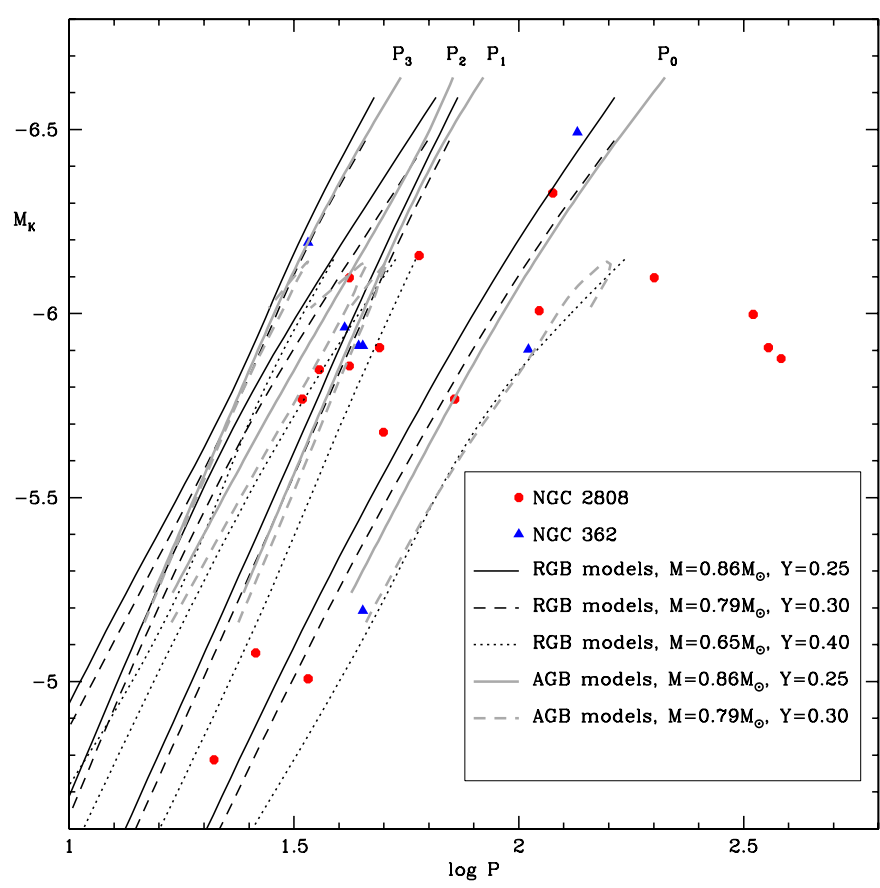

Fig. 12. Same as Fig. 10 but for models with mass loss.

The variables are shown in the $\log P-K$ diagram in Fig. 12. In NGC 2808 most of the fundamental mode pulsators can now be readily explained as RGB or AGB stars with $Y=0.25$ or RGB stars with $Y=0.30$. The star LW3 in NGC 2808 with $\log P \sim 2.3$ now has an interesting explanation as a fundamental mode pulsator at the very tip of the RGB with a high helium abundance of $Y \sim 0.4$, or perhaps as an AGB star with $Y \sim 0.3$. The shorter period of LW3 (42 days) is more consistent with overtone pulsation in the $Y \sim 0.3$ models than with the $Y \sim 0.4$ models. We also note that two of the first overtone pulsators in NGC 2808 have periods suggesting a helium abundance $Y \sim 0.4$.

If the explanation in terms of high helium abundance is correct, it is completely independent evidence for a high helium abundance in some stars in globular clusters. It would be interesting to see if other globular clusters which are estimated to have populations of high helium abundance stars also have LPVs whose periods can only be explained by a high helium abundance. Note that this test can not be used with RR Lyrae stars since the requirement that the RR Lyraes lie in the instability strip confines their helium abundance to a small range for a given cluster age.

In NGC 362 where $Y=0.25$ should be appropriate, the models with mass loss provide a better fit in most cases, although there are two fundamental mode pulsators that appear to require some helium enhancement (to $Y \sim 0.3$ for AGB pulsation or $Y \sim 0.4$ for RGB pulsation).

\section{Conclusions}

We have found and determined pulsation periods for LPVs in the Galactic globular clusters NGC 362 and NGC 2808. By modelling the pulsation of the LPVs, we have shown that mass loss is required on the RGB at a rate that is close to that required to explain the morphology of the horizontal branch. The models show that helium abundance variations of the size estimated from the multiple main-sequences in NGC 2808 lead to a significant increase in the pulsation period of LPVs, especially for the fundamental mode. In NGC 2808, we have found that the spread in fundamental mode periods suggests a spread in helium abundance. One fundamental mode pulsator requires $Y \sim 0.3-0.4$ if its period is to be explained while the periods of two overtone pulsators are also best explained with a helium abundance $Y \sim 0.4$. This is a completely independent line of evidence for a high helium abundance in some stars in globular clusters. Detection and analysis of LPVs in other clusters where helium abundance variations are thought to occur could provide useful confirmation of the high helium abundances.

Acknowledgements. The work of T.L. was funded by the Austrian Science Fund FWF project P20046-N16. The work of P.R.W. was partially funded by Australian Research Council grant DP1095368.

\section{References}

Alard, C. 2000, A\&AS, 144, 363

Alcaino, G. 1976, A\&AS, 26, 359

Alcock, C., Axelrod, T. S., Bennet, D. P., et al. 1992, in Robotic Telescopes in the 1990s, ed. A.V. Fillippenko, ASP Conf. Ser., 34, 193

Bolte, M. 1987, ApJ, 315, 469

Boyer, M., McDonald, I., van Loon, J. Th., et al. 2009, ApJ, 705, 746

Bedin, L. R., Piotto, G., Zoccali, M., et al. 2000, A\&A, 363, 159

Bertelli, G., Girardi, L., Marigo, P., \& Nasi, E. 2008, A\&A, 484, 815

Cacciari, C., Bragaglia, A., Rossetti, E., et al. 2004, A\&A, 413, 343

Caldwell, S. P., \& Dickens, R. J. 1988, MNRAS, 234, 87

Cariulo, P., Degl'Innocenti, S., \& Castellani, V. 2004, A\&A, 421, 1121

Carpenter, J. 2001, AJ, 121, 2851

Carretta, E., \& Gratton, R. G. 1997, A\&AS, 121, 95

Carretta, E., Bragaglia, A., \& Cacciari, C. 2004, ApJ, 610, L25

Carretta, E., Bragaglia, A., Gratton, R. G., et al. 2006, A\&A, 450, 523

Catelan, M., \& de Freitas Pcheco, J. A. 1999, A\&A, 289, 394

Clement, C. M., \& Hazen, M. L. 1989, AJ, 97, 414

Clement, C. M., Muzzin, A., Dufton, Q., et al. 2001, AJ, 122, 2587

Corwin, T. M., Catelan, M., Borissova, J., \& Smith, H. A. 2004, A\&A, 421, 667

Dallesandro, E., Salaris, M., Ferraro, F. R., et al. 2011, MNRAS, 410, 694

D'Antona, F., \& Caloi, V. 2004, ApJ, 611, 871

D'Antona, F., \& Caloi, V. 2008, MNRAS, 390, 693

D'Antona, F., \& Ventura, P. 2008, Msgnr., 134, 18

Eggen, O. J. 1972, ApJ, 172, 639

Fabbri, S., Origlia, L., Rood, R. T., et al. 2008, MemSAI, 79, 720

Ferraro, F. R., Clementini, G., Fusi Pecci, F., Buonanno, R., \& Alcaino, G. 1990 , A\&AS, 84, 59

Fischer, P., Welch, D. L., Mateo, M., \& Coté, P. 1993, AJ, 106, 1508

Frogel, J. A., \& Elias, J. H. 1988, ApJ, 324, 823

Girardi, L., Groenewegen, M. A. T., Hatziminaoglou, E., \& da Costa, L. 2005, A\&A, 436, 895

Gratton, R. G. 1987, A\&A, 179, 181

Gratton, R. G., Fusi Pecci, F., Carretta, E., et al. 1997, ApJ, 491, 749

Green, E. M., \& Norris, J. E. 1990, ApJ, 353, L17

Harris, W. E. 1982, ApJS, 50, 573

Harris, W. E. 1996, AJ, 112, 1487

Höfner, S. 2008, A\&A, 491, L1

Houdashelt, M. L., Bell, R. A., \& Sweigart, A. V. 2000a, AJ, 119, 1448

Houdashelt, M. L., Bell, R. A., Sweigart, A. V., \& Wing, R. F. 2000b, AJ, 119, 1424

Ita, Y., Tanabé, T., Matsunaga, N., et al. 2004, MNRAS, 347, 720

Ita, Y., Tanabé, T., Matsunage, N., et al. 2007, PASJ, 59, 437

Kayser, A., Hilker, M., Grebel, E. K., \& Willemsen, P. G. 2008, A\&A, 486, 437

Lebzelter, T., \& Wood, P. R. 2005, A\&A, 441, 1117

Lebzelter, T., \& Wood, P. R. 2007, A\&A, 475, 643

Lloyd-Evans, T. 1983, MNRAS, 204, 961

Marin-Franch, A., Aparicio, A., Piotto, G., et al. 2009, ApJ, 694, 1498

Mauas, P. J. D., Cacciari, C., \& Pasquini, L. 2006, A\&A, 454, 609

McDonald, I., \& van Loon, J.Th. 2007, A\&A, 476, 1261

McDonald, I., van Loon, J. Th., Dupree, A. K., \& Boyer, M. L. 2010a, MNRAS, 405, 1711

McDonald, I., Sloan, G. C., Zijlstra, A. A., et al. 2010b, ApJ, 717, L92

Meissner, F., \& Weiss, A. 2006, A\&A, 456, 1085

Menzies, J. 1967, Nature, 214, 689

Nicholls, C. P., Wood, P. R., Cioni, M.-R. L., \& Soszyński, I. 2009, MNRAS, 399, 2063

Origlia, L., Ferraro, F. R., Fusi Pecci, F., \& Rood, R. T. 2002, ApJ, 571, 458 
T. Lebzelter and P. R. Wood: Long period variables and mass loss in the globular clusters NGC 362 and NGC 2808

Pilachowski, C. A. 1981, Bull.AAS, 13, 545

Piotto, G., King, I. R., Djorgovski, S. G., et al. 2002, A\&A, 391, 945

Piotto, G., Bedin, L. R., Anderson, J., et al. 2007, ApJ, 661, L53

Reimers, D. 1975, in Problems in Stellar Atmospheres and Envelopes, ed. B.

Bascheck, W. H. Kegel, \& G. Traving (Berlin: Springer), 229

Rieke, G. H., \& Lebofsky, M. J. 1985, ApJ, 288, 618

Rosenberg, A., Aparicio, A., Saviane, I., \& Piotto, G. 2000, A\&AS, 145, 451

Rutledge, G. A., Hesser, J. E., Stetson, P., et al. 1997, PASP, 109, 883

Salaris, M., \& Weiss, A. 2002, A\&A, 388, 492

Sandquist, E. L., \& Martel, A. R. 2007, ApJ, 654, L65

Santos, J. F. C., \& Piatti, A. E. 2004, A\&A, 428, 79

Sawyer, H. B. 1931, Harvard Circular, 366, 1

Schuller, F., Ganesh, S., Messineo, M., et al. 2003, A\&A, 403, 955

Shetrone, M. D., \& Keane, M. J. 2000, AJ, 119, 840

Sloan, G. C., Matsunaga, N., Matsuura, M., et al. 2010, ApJ, 719, 1274
Smith, G. H. 1983, AJ, 88, 410

Smith, G. H. 1984, AJ, 89, 1545

Smith, V. V., Shetrone, M. D., \& Keane, M. J. 1999, ApJ, 516, L73

Soszyński, I., Udalski, A., Szymański, M. K. et al. 2009, Acta Astron., 59, 239 Sperl, M. 1998, Comm. Asteroseism., 111, 1

Szekely, P., Kiss, L. L., Jackson, R., et al. 2007, A\&A, 463, 589

Tucholke, H. J. 1992, A\&AS, 93, 311

Udalski, A., Soszyński, I., Szymański, M. K. et al. 2008, Acta Astron., 58, 329

Walker, A. 1999, AJ, 118, 432

Wood, P. R. 1979, ApJ, 227, 220

Wood, P. R., \& Arnett, D. 2010, ASP Conf. Ser., in press

Wood, P. R., Alcock, C., Allsman, R., et al. 1999, IAU Symp., 191, 151

Wood, P. R., Olivier, E. A., \& Kawaler, S. D. 2004, ApJ, 604, 800

Zacharias, N., Monet, D. G., Levine, S., et al. 2005, Vizier Online Data Catalogue, 1297 